\title{
Single dose of Methotrexate therapy followed by suction curettage for management of Cesarean Scar Pregnancy
}

\section{Abstract}

Aim: To evaluate the efficacy and safety of single dose of of Systemic MTX $50 \mathrm{mg} / \mathrm{m} 2$ followed by D\&S in cases of Cesarean scar pregnancy (CSP).

Objective: Implantation of the pregnancy in a cesarean scar is a rare condition named; Cesarean scar pregnancy (CSP). Maternal complications can be prevented with the early diagnosis and an appropriate management. It is a prospective clinical study to evaluate the efficacy and success rate of single dose use of methotrexate (MTX) followed by dilation and suction $(D \& S)$ regimen in the management of women with cesarean scar pregnancy (CSP).

Methods: 50mg of MTX in the form of a single dose Intramuscular injection then cervical dilatation and suction aspiration with a Karman cannula (D\&S) under guidance of ultrasound after 48 preceded by vaginal misoprostol 2 tablet $(200 \mathrm{mg}) 4$ hours ago.

Results: The mean gestational age at diagnosis was $(8.5 \pm 1.6)$ and the mean gestational age of pregnancy was $8.5 \pm 1.6$. The mean level of serum b-human chorionic gonadotrophin was (7424 \pm 2.560$) .88 .7 \%$ was the successive rate without complication or need of intervention, $2(5.7 \%)$ patients needed intrauterine foley's catheter for 24 hours as a mechanical hemostasis. $2(5.7 \%)$ had laparotomy with wedge resection of the gestational sac lesion and successful repair of the uterine defect and one $(2.8 \%)$ underwent subtotal hysterectomy.

Conclusion: Systemic single dose MTX injection followed by D\&S is an effective and harmless management for CSP. Nevertheless more studies are required to prove the efficiency, safety, and reproductive outcome of variant modalities in treatment of CSP.

Keywords: ectopic pregnancy, cesarean section, scar, curettage, MTX, treatment
Volume 9 Issue 2 - 2020

\author{
Hend S Saleh, Hala E Sherif and Eman M \\ Mahfouz \\ Obstetrics and Gynecology Department, Faculty of Medicine, \\ Zagazig University, Zagazig, Egypt
}

Correspondence: Hend S Saleh, Obstetrics and Gynecology Department, Faculty of Medicine, Zagazig University, Zagazig, Egypt, Email drhendsaleh@yahoo.com

Received: February 21, 2020 | Published: April 20, 2020
Abbreviations: CSP, cesarean scar pregnancy; MTX, methotrexate; HCG, human chorionic gonadotropin; MRI, magnetic resonance imaging

\section{Introduction}

Increasing the incidence cesarean delivery lead to attendance of one serious complication which is Cesarean scar pregnancy (CSP). It is a uncommon form of ectopic pregnancy in which the gestational sac is imbedded in a cesarean scar of the lower uterine segment. CSP is a risky condition, probably leading to immense bleeding uterine rupture, and life-threatening complications. ${ }^{2}$ The accurate incidence of CSP is unidentified. It is presently valued at 1:18002200 pregnancies. It exemplifies $6.1 \%$ of whole ectopic pregnancies with a history of at least one previous cesarean section, ${ }^{3}$ The etiology and pathophysiology of CSP is still unidentified, may be related to a standing scar defect or microscopic dehiscent tract created between the previous cesarean scar tissue and the endometrial canal. ${ }^{4}$ In the early days of pregnancy the blastocyst invades the myometrium via a microscopic abrasion present in the cesarean scar linked to a preceding uterine trauma such as cesarean section, metroplasty, myomectomy, and may be the manual elimination of the placenta. Some authors revealed its potential association to intrauterine Device and pelvic inflammatory disease. ${ }^{5}$ The most common symptom is painless vaginal bleeding that may be massive. However there is no definite clinical sign of CSP.
Clinical history, serial serum human chorionic gonadotropin (HCG) measurements and transvaginal ultrasound examination, mainly in pregnant woman with a previous cesarean delivery early in pregnancy are necessary for early diagnosis and termination of that pregnancy. ${ }^{6}$ Also magnetic resonance imaging (MRI), and endoscopic modalities may be helpful for diagnosis and the management of (CSP). It is frequently required for cases in which the TVUS is not definite or did not obviously prove urinary bladder Envelopment. ${ }^{7}$ To decrease the threat of a false diagnosis and improve its accuracy, a collective ultrasound (TVS, TAS, color flow Doppler, and three-dimensional TVS) should be suggested. ${ }^{8}$ The modalities of treatment are medical, surgical or combined. There is no agreement on the favorite mode of management. Medical protocol Includes systemic (single or repeated doses) or local administration of methotrexate (MTX), potassium chloride, trichosanthes, or mifepristone. ${ }^{9}$ Surgical options; embrace uterine curettage, hysteroscopic resection, laparotomy or laparoscopic resection for patients are wishing to reserve fertility. ${ }^{10}$ Selection of mode of termination depends on features like size of pregnancy, the haemodynamic Prominence of the patient, presence or absence of scar rupture, levels of hCG, and craving for upcoming fertility. ${ }^{11}$ MTX is an anti metabolite drug used broadly in treatment of ectopic pregnancies. Systemic route is the least invasive management and has been commonly used for stable patients. Fertility preservation and reducing the requirement to surgery are the main advantages of its use. However, its use alone needs an extended time to follow-up both 
beta-hCG to return to normal and gestational mass to resolve ${ }^{9}$ Some studies proved that CSP responded well to the single dose of Systemic MTX $50 \mathrm{mg} / \mathrm{m} 2$ when HCG level is lower than $5000 \mathrm{mIU} / \mathrm{ml} .{ }^{12}$ Others found that single-dose, systemic MTX was not sufficient, so they had to achieve multiple doses of MTX with its drawbacks. ${ }^{13}$ Others found that combination of single dose of systemic Methotrexate followed by D\&S can avoid these needless laparotomy and preserve fertility in most women with $\mathrm{CSP}^{15}$

\section{Material and methods}

This is a prospective clinical study was done on 35 pregnant females with a diagnosis of CSP between 6 and 11weeks were admitted to our department from January 2017 to July 2019. Gestational age was considered built on last menstrual period and accustomed according to the ultrasound dating. They were managed by MTX injection followed by D\&S (combined therapy group). All enrolled Women were hemodynamically stable, had no internal bleeding, or ruptured CSP, the gestational sac \pm 8 weeks and had no contraindications to MTX, like elevated liver neutropenia or disturbed renal function tests. The diagnosis of CSP was proved according to the following criteria;

i. Positive serum b-hCG levels,

ii. History of lower uterine segment cesarean delivery

iii. Gratification of the following ultrasonographic conditions;

a) visualized endometrium with an empty uterine cavity

b) a pure observable empty cervical canal;

c) A gestational sac with or without cardiac activity positioned anteriorly at the level of the the lower uterine segment with cesarean scar, \{internal os $\}$ inside a evident myometrial fault between the bladder and the sac on sagittal view of the uterus.

d) Negative "sliding organs sign," which was demarcated as the failure to dislodge the gestational sac from its place at the level of the internal os using mild pressure smeared by the transvaginal probe.

e) Suggestion of functional placental circulation/trophoblastic on color flow Doppler examination ${ }^{4}$

A written informed consent was taken from all participants. Our study was approved by the institutional research ethical committee of zagazig University according the standards of Helsinki Declaration. Full information's and counseling about nature of management and its hazards were given to the patients. All patients were managed by; $50 \mathrm{mg}$ of MTX in the form of a single dose Intramuscular injection then cervical dilatation and suction aspiration with a Karman cannula (D\&S) under guidance of ultrasound after 48 preceded by vaginal misoprostol 2 tablet $(200 \mathrm{mg}) 4$ hours ago. Positive outcomes were:

- Decreasing serum b-hCG levels up to normal level.

- Vanishing CSP mass,

- Evading the foremost complications like; rupture of uterine scar, hemorrhage, conversion to laparoscopic surgery or laparotomy, or hysterectomy. Statistical analyses were done with SPSS for Windows (version 16.0; SPSS, Chicago, IL). Data were analyzed for normal distribution with the Kolmogorov-Smirnov test and for homogeneity of variance with Levene test. The variables did not meet homogeneity of variance and normality and were analyzed using Mann-Whitney U test.

\section{Results}

The demographic criteria of patients were presented in Table 1. Mean age, parity and gravity of patients was ( $32.1 \pm 3.5$ years), $2.1 \pm 0.5$ and $3.4 \pm 1.7$ respectively. At ultrasound scan, all 35 women had an empty uterine cavity With the gestational placed at the site of scar, nearby the bladder. (Figure 1,2) All women had a history of previous cesarean section. The mean gestational age at diagnosis $8.5 \pm 1.6$ (wks). The mean Levels of HCG were documented before starting the management $7424 \pm 2.560(\mathrm{mIU} / \mathrm{mL})$. The mean of myometrial thickness between the sac and the bladder wall under ultrasonic investigation was $2.6 \pm 0.89 \mathrm{~mm}$. All females had a history of previous uterine surgery. The mean number of previous cesarean sections was $3.6 \pm 0.72$, from the 35 women, $8(22.5 \%)$ had three, $18(51.4 \%)$ had two, $6(17.1 \%)$ had one, $2(5.7 \%)$ had had four and $1(2.8 \%)$ had five CDs.

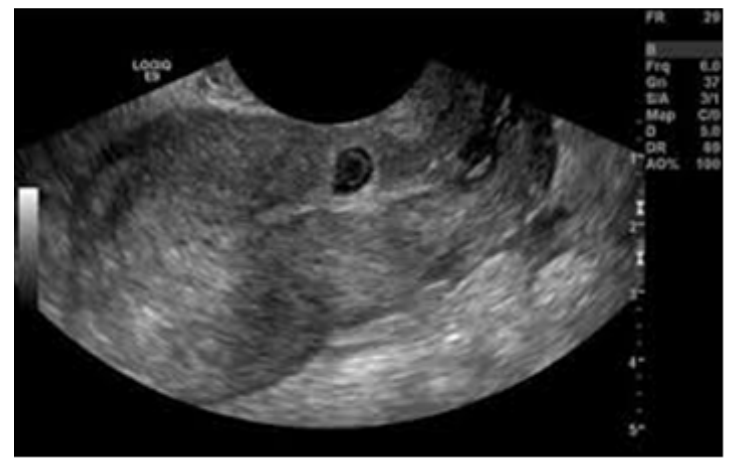

Figure I Transabdominal ultrasonography showed, a scar ectopic pregnancy.

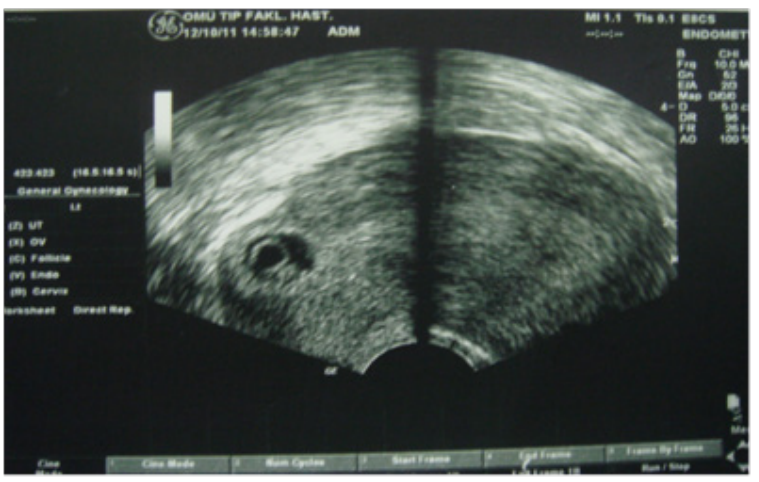

Figure 2 Transvaginal sonography showed, empty uterine cavity with a gestational sac at the lower-anterior wall of previous scarred uterus.

Table I Demographic criteria of patients at diagnosis

\begin{tabular}{ll}
\hline Parameter & No. 35 \\
\hline Age (ys) & $32.1 \pm 3.5$ \\
Gestational age (wks) & $8.5 \pm 1.6$ \\
Gravidity & $3.4 \pm 1.7$ \\
Parity & $2.1 \pm 0.5$ \\
Previous c.s & $3.6 \pm 0.72$ \\
Myometrial thickness & $2.6 \pm 0.89$ \\
B-HCG (mlU/mL) & $7424 \pm 2.560$ \\
Number of cases with & \multicolumn{1}{c}{13} \\
cardiac pulsation & $37 \%$
\end{tabular}

Values are presented as mean \pm SD, No. (Percentage \%) 
Table 2 Clinical outcome after management

\begin{tabular}{|c|c|c|}
\hline Estimated blood loss ( $\mathrm{mL}$, mean $\pm \mathrm{SD}$ & \multicolumn{2}{|c|}{$698.8 \pm 937.2$} \\
\hline Success rate without complication & 31 & $88.6 \%$ \\
\hline $\begin{array}{l}\text { Resolution time of serum b-hCG } \\
\text { (days, median [range]) }\end{array}$ & \multicolumn{2}{|c|}{21 days $\{17-60\}$} \\
\hline $\begin{array}{l}\text { Bleeding need intra uterine folys } \\
\text { catheter }\end{array}$ & 2 & $5.7 \%$ \\
\hline Laparotomy & 3 & $8.5 \%$ \\
\hline $\begin{array}{l}\text {-Hystrotomy with wedge resection } \\
\text { and repair }\end{array}$ & 2 & $5.7 \%$ \\
\hline - Hysterectomy & I & $2.8 \%$ \\
\hline
\end{tabular}

Values are presented as mean \pm SD, No. (Percentage \%)

At the time of diagnosis, $50 \%$ of the females were complaining from mild vaginal bleeding, and remaining was diagnosed with routine antenatal ultrasound examination without complaint.

Post dilatation and suction (D\&S), two cases had plentiful vaginal bleeding which was controlled with a Foley's catheter put intrauterine for 24 hours as a mechanical hemostasis. Three patients need laparotomic hysterotomy two of them can managed by wedge resection of the gestational sac lesion and successful repair of the uterine defect. Only one had profuse intraoperative bleeding and big defect in the uterus at the scar area, which was controlled by urgent subtotal hysterectomy, salpingectomy, with conservation of the ovaries. No more complications postoperatively were observed either at the 1-week or 1-month follow-up.

\section{Discussion}

Systemic administration of MTX is a standard management for tubal and cervical pregnancy. ${ }^{15}$ In the Current study we found that a single dose of $50 \mathrm{mg}$ IM MTX followed by D\&S had a high cure rate, In patients with CSPs. In study of Hua Wang, et al., ${ }^{5}$ who compared the efficacy of methotrexate (MTX) regimen only or MTX regimen followed by dilation and curettage (D\&C). in women with cesarean scar pregnancy (CSP), they found that Both regimens could treat most of CSP patients efficaciously, but the combined one caused a shorter period of treatment and designated a more satisfactory effect. ${ }^{16}$ In the current study, $88.6 \%(N=31)$ of patients with CSP were managed successfully without any complications. 4 cases $(11.4 \%)$ needed interventions, 2 cases had plentiful vaginal bleeding which was controlled with a Foley's catheter was put intrauterine for 24 hours as a mechanical hemostasis, 3 were underwent laparotomic hysterotomy, two of them can managed by wedge resection and repair of the uterine defect. Only one was had subtotal hysterectomy, salpingectomy, with conservation of the ovaries. Timor-Tritsch and Monteagudo ${ }^{16}$ investigated the dissimilar therapeutic lines of the CSP. They found that, the highest number of complications were those involving the use of MTX alone (62\%), D\&C (62\%), UAE (47\%), and the administration of intramuscular MTX combined with D\&C (86\%). In our practice, utmost of the females were cured with The combined line of MTX and D\&S. Giampaolino et al., ${ }^{14}$ established in their study that maximum number of the patients were treated effectively and safely with the combined line of MTX and D\&S or UAE and D\&S with no complications as the success rate was $100 \%$.This agreed with our results but was in dissimilarity to previously reported data in the study of Timor-Tritsch and Monteagudo ${ }^{16}$ may be due to the selection mode of the patient as Giampaolino et al., ${ }^{14}$ limited their management to females with earlier gestational periods. ${ }^{17}$ Lian et al. ${ }^{18}$ measured the efficiency of using treatment of single dose $50 \mathrm{mg}$ systemic MTX followed by embolization of uterine artery and then administration of MTX injection locally if the b-hCG levels did not decline to the half of the primary level. They attained a $57.1 \%$ success rate with systemic MTX, and the residual patients were cured with UAE and local MTX injection.

Cheung et al. ${ }^{19}$ demonstrated that the addition of intramuscular MTX to local one increased the cure rate to $88.5 \%$. Uludag et al. ${ }^{20}$ found that both local and systemic MTX treatments were in the same effect in handling of CSP with full success. But established that conventional treatment and follow-up protocols of local and systemic MTX should be revised. So, from all revision to lots of studies in the same literature, the standard handling line has not been proven in the managing of scar pregnancy yet. Nevertheless, the accurate diagnosis and the modified evaluation of risk factors could funding physicians in creating the best choice of effectiveness and safety.

\section{Conclusion}

The rarest form of ectopic pregnancies is cesarean scar ectopic pregnancy (CSP). It could lead to severe complications. So, intensified awareness must be in consideration for both early diagnosis and management to prevent serious catastrophe. Systemic single dose MTX injection followed by D\&S is an effective and harmless management for CSP. Nevertheless more studies are required to prove the efficiency, safety, and reproductive outcome of variant modalities in treatment of CSP.

\section{Ethical approval}

The work conforms to the provisions of the Declaration of Helsinki.

\section{Conflicts of interest}

No potential conflicts of interest were reported by the authors.

\section{References}

1. Rotas MA, Haberman S, Levgur M. Cesarean scar ectopic pregnancies: etiology, diagnosis, and management. Obstet Gynecol. 2006;107(6):13731381.

2. Seow KM, Huang LW, Lin YH, et al. Cesarean scar pregnancy: issues in management. Ultrasound Obstet Gynecol. 2004;23(3):247-253.

3. Yela DA, Marchiani N. Conservative management of ectopic pregnancy in cesarean scar: case report. Rev Bras Ginecol Obstet. 2013;35:233-237.

4. Jurkovic D, Hillaby K, Woelfer B, et al. First-trimester diagnosis and management of pregnancies implanted into the lower uterine segment Cesarean section scar. Ultrasound Obstet Gynecol. 2003;21(3):220-227.

5. Wang JH, Wu RJ, Xu KH, et al. Single large cystic adenomyoma of the uterus after cornual pregnancy and curettage. Fertil Steril. 2007;88(4):965-967.

6. Ash A, Smith A, Maxwell D Caesarean scar pregnancy. $\mathrm{Br} J$ Obstet Gynaecol. 2007;114(3):253-263.

7. Wu R, Klein MA, Mahboob S, et al. Magnetic resonance imaging as an adjunct to ultrasound in evaluating cesarean scar ectopic pregnancy. $J$ Clin Imaging Sci. 2013;3:16.

8. Wang CJ, Chao AS, Yuen LT, et al. Endoscopic management of cesarean scar pregnancy. Fertil Steril. 2006;85(2):494.e1-4.

9. Wang HY, Zhang J, Li YN, et al. Laparoscopic management or laparoscopy combined with transvaginal management of type II cesarean scar pregnancy. JSLS. 2013;17(2):263-272. 
10. Tagore S, Teo SH, Chua SY, et al. A retrospective review of uterine scar pregnancies: single centre experience. Arch Gynecol Obstet. 2010;282(6):711-715.

11. Michener C, Dickinson JE. Caesarean scar ectopic pregnancy: a single centre case series. Aust N Z J Obstet Gynaecol. 2009;49(5):451-455.

12. Maymon R, Halperin R, Mendlovic S, et al. Ectopic pregnancies in a Caesarean scar: review of the medical approach to an iatrogenic complication. Hum Reprod Update. 2004;10(6):515-523.

13. Muraji M, Mabuchi S, Hisamoto K, et al. Cesarean scar pregnancies successfully treated with methotrexate. Acta Obstet Gynecol Scand. 2009;88(6):720-723.

14. Giampaolino P, N. De Rosa, Morra I, et al. Management of Cesarean Scar Pregnancy: A Single-Institution Retrospective Review. BioMed Research International. 2018; ID 6486407, 9.

15. Huang TH, Shau WY, Hsieh TT, et al. Prognostic factors for an unsatisfactory primary methotrexate treatment of cervical pregnancy: a quantitative review. Hum Reprod. 1998;13(9):2636-2642.
16. Timor-Tritsch IE, Monteagudo A. Unforeseen consequences of the increasing rate of cesarean deliveries: early placenta accreta and cesarean scar pregnancy. A review. Am J Obstet Gynecol. 2012; 207(1):14-29.

17. Cerveira I, Costa C, Santos F, et al. Cervical ectopic pregnancy successfully treated with local methotrexate injection. Fertility and Sterility. 2008;90(5):2005-e10.

18. Lian F, Wang Y, Chen W, et al. Uterine artery embolization combined with local methotrexate and systemic methotrexate for treatment of cesarean scar pregnancy with different ultrasonographic pattern. Cardiovasc Intervent Radiol. 2012;35(2):286-291.

19. Cheung VY. Local methotrexate injection as the first-line treatment for cesarean scar pregnancy: review of the literature. J Minim Invasive Gynecol. 2015;22(5):753-758.

20. Semih Z. Uludag, Mehmet S. Kutuk, Mehmet Ak, et al. Comparison of systemic and local methotrexate treatments in cesarean scar pregnancies \& Gynecology and Reproductive Biology. European Journal of Obstetrics \& Gynecology and Reproductive Biology. 2016;206:131-135. 